\title{
Loeys-Dietz and Shprintzen-Goldberg syndromes: analysis of TGF- $\beta$-opathies with craniofacial manifestations using an innovative multimodality method
}

\author{
Konstantinia Almpani (D) , 'Denise K. Liberton, ${ }^{1}$ Priyam Jani (D) , 'Cyrus Keyvanfar, ${ }^{1}$ \\ Rashmi Mishra, ${ }^{1}$ Natasha Curry, ${ }^{1}$ Pamela Orzechowski, ${ }^{1}$ \\ Pamela A. Frischmeyer-Guerrerio, ${ }^{2}$ Janice S. Lee ${ }^{1}$
}

- Additional supplemental material is published online only. To view, please visit the journal online (http://dx. doi.org/10.1136/jmedgenet2021-107695)

${ }^{1}$ Craniofacial Anomalies and Regeneration Section, National Institute of Dental and Craniofacial Research, Bethesda Maryland, USA

${ }^{2}$ Food Allergy Research Unit, National Institute of Allergy and Infectious Diseases, $\mathrm{NIH}$ Bethesda, Maryland, USA

\section{Correspondence to}

Dr Janice S. Lee, Craniofacial Anomalies and Regeneration Section, National Institute of Dental and Craniofacial Research, Bethesda, MD 20892 USA; janice.lee@nih.gov

Received 4 January 2021 Accepted 2 October 2021

\section{Check for updates}

(c) Author(s) (or their employer(s)) 2021. Re-use permitted under CC BY-NC. No commercial re-use. See rights and permissions. Published by BMJ.

\section{To cite: Almpani $\mathrm{K}$,}

Liberton DK, Jani $\mathrm{P}$, et al.

$J$ Med Genet Epub ahead of print: [please include Day

Month Year]. doi:10.1136/

jmedgenet-2021-107695

\begin{abstract}
Background Elevated transforming growth factorbeta (TGF- $\beta$ ) signalling has been implicated in the pathogenesis of Loeys-Dietz syndrome (LDS) and Shprintzen-Goldberg syndrome (SGS). In this study, we provide a qualitative and quantitative analysis of the craniofacial and functional features among the LDS subtypes and SGS.
\end{abstract}

Methods We explore the variability within and across a cohort of 44 patients through deep clinical phenotyping, three-dimensional (3D) facial photo surface analysis, cephalometric and geometric morphometric analyses of cone-beam CT scans.

Results The most common craniofacial features detected in this cohort include mandibular retrognathism $(84 \%)$, flat midface projection (84\%), abnormal eye shape (73\%), low-set ears (73\%), abnormal nose $(66 \%)$ and lip shape $(64 \%)$, hypertelorism $(41 \%)$ and a relatively high prevalence of nystagmus/strabismus $(43 \%)$, temporomandibular joint disorders $(38 \%)$ and obstructive sleep apnoea (23\%). 3D cephalometric analysis demonstrated an increased cranial base angle with shortened anterior cranial base and underdevelopment of the maxilla and mandible, with evidence of a reduced pharyngeal airway in $55 \%$ of those analysed. Geometric morphometric analysis confirmed that the greatest craniofacial shape variation was among patients with LDS type 2, with distinct clustering of patients with SGS.

Conclusions This comprehensive phenotypic approach identifies developmental abnormalities that segregate to mutation variants along the TGF- $\beta$ signalling pathway, with a particularly severe phenotype associated with TGFBR2 and SKI mutations. Multimodality assessment of craniofacial anomalies objectively reveals the impact of mutations of the TGF- $\beta$ pathway with perturbations associated with the cranium and cranial base with severe downstream effects on the orbit, maxilla and mandible with the resultant clinical phenotypes.

\section{INTRODUCTION}

Mutations of the transforming growth factor-beta (TGF- $\beta$ ) signalling pathway have been shown to play a critical role in the aetiology of several connective tissue disorders including Loeys-Dietz syndrome (LDS) and Sphrintzen-Goldberg syndrome (SGS). LDS (types 1-5, MIM\# 609192, $610168,613795,614816,615582)$ is a rare autosomal dominant connective tissue disorder with multisystemic involvement, resulting from heterozygous mutations in the TGF- $\beta$ signalling pathway. ${ }^{1}$ Originally, LDS was misdiagnosed as Marfan syndrome (FBN1) due to similar vascular features, including aortic root aneurysms. However, patients with LDS have more severe cardiac symptoms, with dissections occurring at younger ages and at smaller diameters throughout the arterial tree, as well as craniofacial abnormalities that have not been thoroughly described.

Five LDS-causing mutations have been identified along the TGF- $\beta$ pathway including the genes encoding TGF- $\beta$ receptor 1 (TGFBR1) and TGF- $\beta$ receptor 2 (TGFBR2), mothers against decapentaplegic homologue 3 (SMAD3), TGF- $\beta$ ligand 2 (TGFB2) and TGF- $\beta$ ligand 3 (TGFB3), considered LDS types 1-5, respectively. ${ }^{2}$ Mutations in the gene encoding the SKI proto-oncogene (SKI), also involved in TGF- $\beta$ signalling through the inhibition of SMADs, have been associated with SGS, a condition with less severe cardiac defects than LDS but more pronounced craniofacial abnormalities. ${ }^{3-5}$

Phenotypically, LDS was originally characterised by a triad of features: arterial tortuosity and aneurysms, hypertelorism, and bifid uvula or cleft palate. ${ }^{6}$ In the decade since its discovery, intense research has been performed on patients and mouse models to better understand the striking variable phenotype of LDS, particularly visible in the craniofacial region. However, not all patients with LDS possess the craniofacial features of the original triad. $^{78}$ Additional craniofacial features reported include craniosynostosis, facial asymmetry, blepharoptosis, retrognathia, malar hypoplasia, dolichocephaly, blue sclerae and strabismus. ${ }^{9-12}$ Previous papers reported that patients with LDS type 2 have mild craniofacial involvement, while patients with LDS type 1 have more significant craniofacial involvement. ${ }^{613}$ However, the assignment of disease type at the time was based on clinical findings, not molecular diagnosis.

Despite the prevalence of craniofacial features in patients with LDS and the high phenotypic 
variability, no study has focused on the disparate craniofacial anomalies and the correlation of genotype to the craniofacial phenotype of the different LDS types, and none has done so quantitatively. The goal of this study is to provide a comprehensive phenotypic characterisation of the craniofacial anomalies of LDS and SGS and explore the variability within and across the different subtypes. This is achieved through deep clinical phenotyping, three-dimensional (3D) facial surface analysis, cephalometric and multivariate geometric morphometric analyses of one of the largest LDS cohorts. Through this comprehensive and quantitative approach, we elucidate the impact of TGF- $\beta$ pathway mutations on craniofacial development in these two rare disorders.

\section{METHODS}

\section{Participants}

Patients with a genetic diagnosis of LDS or SGS were enrolled at the National Institutes of Health (NIH) Clinical Center between 2015 and 2018. Participants were consented onto Institutional Review Board-approved protocols (NCT02639312, Principal Investigator: Lee; NCT02504853, Principal Investigator: Guerrerio). Examinations and imaging were conducted at the $\mathrm{NIH}$ Dental Clinic.

\section{Clinical evaluation}

The craniofacial anomalies team of the National Institute of Dental and Craniofacial Research (NIDCR) performed a comprehensive craniofacial examination which included a clinical and anthropometric 120-point assessment of the face, skull, neck and temporomandibular joint (TMJ). The orodental phenotype is described elsewhere. ${ }^{14}$ The Oral Health Impact Profile 14 and self-reported NIDCR-25 questionnaires were completed; the results have been previously published. ${ }^{15}$ Relevant craniofacial findings and functional deficits, such as temporomandibular disorder (TMD), snoring, obstructive sleep apnoea (OSA), hearing loss, and strabismus/nystagmus were included in this study. Surgical procedures related to the craniofacial region were documented.

\section{Craniofacial Anomalies Index}

A craniofacial clinical phenotype and functional abnormalities index (Craniofacial Anomalies Index (CAI)) was developed to aid in the correlation to genotype (table 1). Each phenotypic or functional characteristic received 1 point when present. The total achievable score was 24 . The average participant scores were calculated for each genotype and used for intergroup comparisons.

\section{Imaging}

Photos

Two-dimensional facial photos were obtained for each patient (Canon EOS 5D Mark II, Canon, USA), including six views: frontal, smiling, lateral left and right, submental and 'bird's eye'. They were used for the validation of clinical findings.

The 3D photos were obtained from 39 patients using the $3 \mathrm{dMD}$ two-pod system (Atlanta, Georgia, USA) or Canfield Vectra Handheld imaging system (Parsippany, New Jersey, USA). ${ }^{16}$ Each individual was captured with a neutral expression, mouth closed and teeth in light contact. The images were automatically stitched using respective proprietary software and exported in Wavefront OBJ (.obj) format with texture. No surface smoothing or hole filling was conducted, and quality checks were performed to ensure uniform stitching. Images were imported into MeshLab, ${ }^{17}$ where 24 landmarks were manually annotated by a trained observer (CK, KA). Facial landmarks and normative measurements were obtained from FaceBase 3D Facial Norms (www.facebase.org, NIDCR Project 1U01DE020078). ${ }^{18}$ Landmark coordinates were exported in .csv format and 29 linear distances were measured. In nine cases, landmark tragion was missing, and the coordinates were imputed via thin-plate spline interpolation using $\mathrm{R}$ software. ${ }^{19}$ In cases with a slightly open mouth $(n=12)$, landmark stomion was replaced with an upper lip stomion to calculate upper facial height and lip measurements, and a lower lip stomion to calculate lower lip measurements. Total and lower facial height measurements were calculated by subtracting the interlabial gap measurements. In all open mouth cases, the lower facial depth measurements were considered unaffected, due to the relatively small magnitude of the mouth opening and lack of condylar translation.

Linear distances were compared with the appropriate sexmatched and age-matched norms to calculate a Z-score, which is the number of SDs from the norm. A Z-score of \pm 2 or greater was considered a clinically significant alteration of facial features; a Z-score between \pm 1 and \pm 2 was considered a subclinical alteration. Normative data are only available for individuals of European/Caucasian ancestry and for individuals from 3 to 40 years of age.

\section{Cone-beam CT scans and cephalometric analysis}

Full-head cone-beam CTs (CBCTs) were obtained when clinically indicated, for a sample size of 20 patients. Planmeca ProMax 3D system (Planmeca, Helsinki, Finland) was used on low dose mode, $400 \mu \mathrm{m}$ resolution. CBCTs were exported in dicom format and cephalometric analysis was performed in Invivo V.5.4 software (Anatomage, San Jose, California, USA). Linear distances and angles were calculated in 3D and compared with age, sex and ethnic-appropriate cephalometric norms (online supplemental table 1). Two patients missing the landmark menton had related measurements omitted. The results are presented as Z-scores. We also tested for significant craniofacial shape differences among the LDS subtypes. LDS1 and LDS2 subtypes were more common, thus, we combined all other types (LDS3, LDS4, LDS5) into a single category for better sample size comparisons.

Quantitative airway space was measured with the automated airway space module in Invivo V.5.4 software. Total volume of the pharyngeal airway space (PAS), minimum cross-sectional area (CSA) and distance of the hyoid bone from mandibular plane (H-MP) were measured.

\section{Multivariate geometric morphometric analysis}

Multivariate geometric morphometric analysis was performed to explore overall patterns and variation in craniofacial shape across the entire LDS cohort. The analyses were based on the landmark coordinate data and performed separately for the CBCT and 3D photo datasets. Established 3D landmarks were used for the CBCTs. ${ }^{20}$ Landmark coordinate data were first imported into $\mathrm{R}$ software and analysed via the Geomorph package. ${ }^{19}{ }^{21}$ Missing landmarks were imputed via thin-plate spline interpolation. The complete set of landmark coordinates was imported into MorphoJ $^{22}$ for analysis and registered via Procrustes superimposition. We tested for associations between craniofacial shape with age and size, and after regressing out these effects, focused on the Procrustes residuals for final analysis. A principal component analysis (PCA) was performed to examine craniofacial variation across LDS types. 


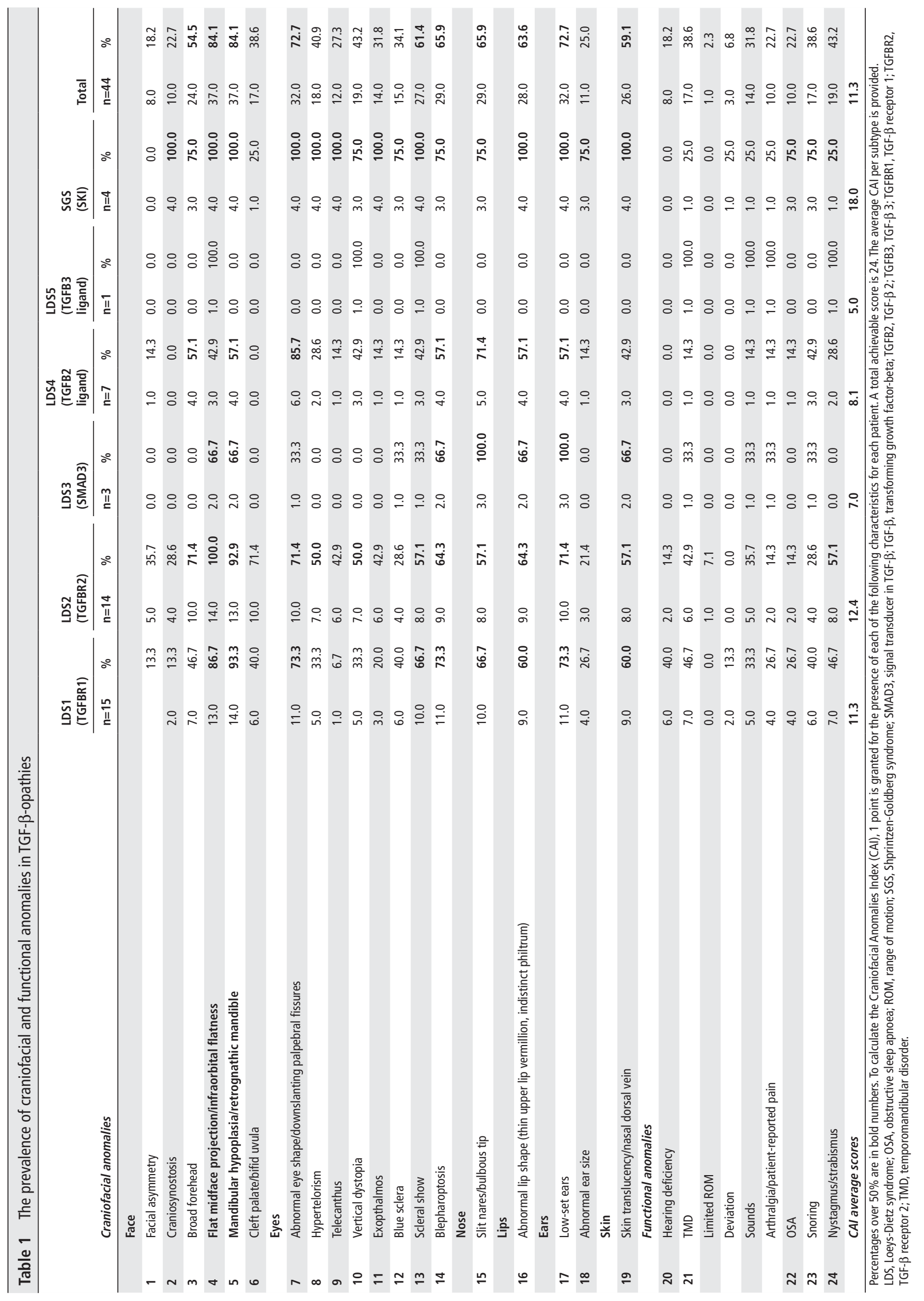




\begin{tabular}{lllc}
\hline Table 2 & Demographics & & \\
\hline & \multirow{2}{*}{$\begin{array}{l}\text { Number of patients } \\
\text { Characteristics }\end{array}$} & \multicolumn{2}{l}{ Age at first visit (years) } \\
\cline { 3 - 4 } Mean \pm SD & Range \\
\hline Total subjects & $44(100)$ & $17.2 \pm 14.6$ & $2.4-57.4$ \\
Gender & & & \\
\hline Male & $22(50)$ & $16.7 \pm 15.6$ & $2.4-55.0$ \\
\hline Female & $22(50)$ & $17.7 \pm 13.9$ & $2.4-57.4$ \\
Race-ethnicity & & & \\
\hline White-Caucasian & $35(79.5)$ & $17.2 \pm 14.1$ & $2.4-55$ \\
\hline Hispanic & $4(9.0)$ & $9.1 \pm 3.5$ & $5.9-13.3$ \\
\hline Black-African American & $2(4.5)$ & $14.9 \pm 3.3$ & $12.6-17.3$ \\
\hline Asian & $2(4.5)$ & $32.2 \pm 35.5$ & $7.1-57.4$ \\
\hline Pathogenic gene mutations & & & \\
\hline TGFBR1 (LDS1) & $15(34)$ & $17.4 \pm 15.2$ & $3.7-55.0$ \\
\hline TGFBR2 (LDS2) & $14(31.8)$ & $18.1 \pm 15.5$ & $3.2-57.4$ \\
\hline SMAD3 (LDS3) & $3(6.8)$ & $19.7 \pm 15.9$ & $3.8-35.5$ \\
\hline TGFB2 (LDS4) & $7(15.9)$ & $16.8 \pm 17.2$ & $2.4-39.8$ \\
\hline TGFB3 (LDS5) & $1(2.3)$ & $37.5 \pm n / a$ & $n / a$ \\
\hline SKI (SGS) & $4(9)$ & $8.4 \pm 2.3$ & $5.9-10.6$ \\
\hline
\end{tabular}

n/a=noted for range and SD with sample size of 1.

LDS, Loeys-Dietz syndrome; SMAD3, signal transducer in transforming growth factor-beta; TGFB2, transforming growth factor-beta 2; TGFB3, transforming growth factor-beta 3; TGFBR1, transforming growth factor-beta receptor 1; TGFBR2, transforming growth factor-beta receptor 2 .

\section{Statistical methods}

All clinical data were collected using REDCap, a web-based platform. ${ }^{23}$ IBM SPSS 28.0 (IBM, USA) and Microsoft Excel were used for generating tables. Descriptive statistics were used, and all data were summarised by means (ie, mean Z-score).

Analysis of variance (ANOVA) was used to compare LDS1, LDS2 and other LDS types for the cephalometric measurements. $\mathrm{P}<0.05$ was considered statistically significant.

\section{RESULTS}

\section{Clinical phenotyping, $\mathrm{N}=44$}

A total of 44 patients, 40 with LDS and 4 with SGS, were enrolled. Among the 40 patients with LDS, 15 had mutations in TGFBR1 (LDS1), 14 had mutations in TGFBR2 (LDS2), 3 had mutations in SMAD3 (LDS3), 7 had mutations in TGFB2 (LDS4) and 1 had a mutation in TGFB3 (LDS5). Mean age was 17.2 years (2.4-57.4 years); 22 were female, 22 were male (table 2 ). Additional information regarding the genetic mutations of the subjects included in this cohort can be found in online supplemental table 2. We also explored the possibility of specific genotype-phenotype correlations using the known genetic variants, with negative results.

Facial shape was highly variable. Craniosynostosis was reported in 10 cases (22.7\%), with the sagittal and metopic sutures affected evenly. Eight patients had clinically significant overall facial asymmetry (18.2\%). However, there were specific regions with asymmetry: $43.2 \%$ with vertical eye dystopia, $20.5 \%$ with deviation of nasal tip, $18.2 \%$ with lip asymmetry. Mandibular hypoplasia or retrognathism (84.1\%) and midface and/or infraorbital flatness (84.1\%) were the most prevalent characteristics. A tall and broad forehead (54.5\%) was common (table 1, figure 1).

The eye shape and position demonstrated downslanting palpebral fissures or other abnormal eye shape (72.7\%), blepharoptosis (65.9\%), increased scleral show (61.4\%), hypertelorism (40.9\%) and telecanthus (27.3\%). Nasal anomalies included

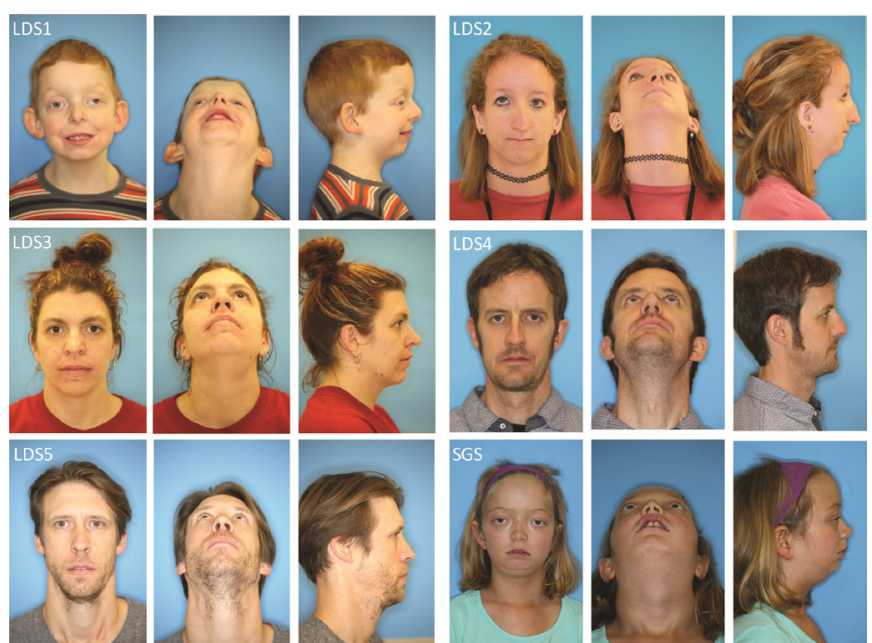

Figure 1 Clinical views of patients with LDS (subtypes 1-5) and SGS. This photo gallery illustrates the highly variable clinical craniofacial phenotype in TGF- $\beta$-opathies. LDS, Loeys-Dietz syndrome; SGS, ShprintzenGoldberg syndrome; TGF- $\beta$, transforming growth factor-beta.

bulbous nasal tip and/or slit-nares shape (65.9\%). Lip anomalies included thin upper lip vermillion or indistinct philtrum $(63.6 \%)$. Ear shape was not affected, but the position of the ears was low set $(72.7 \%)$. The presence of a submucous cleft palate was confirmed in only two cases, while bifid uvula was more common (38.6\%). There was no cleft lip or palate. A nasal dorsal vein was characteristic in $38.6 \%$, as well as velvet, translucent skin texture (59.1\%).

\section{LDS subtype distinctions}

SGS and LDS2 had the highest percentage of craniofacial anomalies. Mandibular retrognathism was the most prevalent among the different subtypes: SGS (100.0\%), LDS1 (93.3\%), LDS2 (92.9\%), LDS3 (66.7\%) and LDS4 (57.1\%). Midface flatness was also common: LDS2 (85.7\%), SGS (75.0\%) and LDS1 (73.3\%). LDS2 (71.4\%) and LDS1 (40.0\%) had palatal deformities (submucosal cleft palate or bifid uvula), while other LDS types did not have submucous cleft or bifid uvula. More patients with LDS2 presented with telecanthus (42.9\%) and hypertelorism $(50 \%)$ compared with the other LDS types. SGS had a very high occurrence $(>75 \%)$ of almost every craniofacial anomaly, including craniosynostosis $(100 \%)$, with the exception of submucous cleft palate/bifid uvula (25\%) (table 1).

\section{Functional abnormalities}

The total prevalence of TMD was $38.6 \%$, which is relatively high considering the young average age of the cohort. The disorders included arthralgia or pain reported by questionnaire (22.7\%), joint sounds $(31.8 \%)$, deviation of the mandible with opening $(6.8 \%)$ and limited range of motion $(2.3 \%)$. Conductive hearing loss was noted in five patients $(11.4 \%)$, and subclinical hearing loss in three patients. Importantly, $22.7 \%$ were diagnosed with OSA and $38.6 \%$ reported snoring. Functional eye abnormalities such as nystagmus and strabismus affected $43.2 \%$ and were most notable in LDS2 (table 1).

\section{CAl scores $(\mathrm{N}=44)$}

Based on the average index scores, SGS had the most craniofacial anomalies among the cohort, with a mean score of 18.0 (range 17.0-19.0) followed by LDS2 $=12.4$ (range 4.0-18.0), LDS1=11.3 (range 3.0-20.0), LDS4=8.1 (range 4.0-11.0), 
LDS3 $=7$ (range 5-8), while the single proband with LDS5 had a score of 5 (table 1).

\section{Surgeries of the craniofacial region}

Fifty-nine per cent of patients had a history of one or more surgeries of the craniofacial region. Among the 10 patients with a diagnosis of craniosynostosis, 6 had a history of surgical repair $(S G S=3 ; \operatorname{LDS} 2=2$; $\operatorname{LDS} 1=1)$. Other reported surgical procedures included adenoidectomy $(n=9)$, tonsillectomy $(n=7)$, tympanostomy tube placement $(\mathrm{n}=8)$, strabismus correction $(n=6)$, and soft palate or bifid uvula repair $(n=5)$. One patient with SGS required mandibular distraction osteogenesis for congenital micrognathia. Other isolated procedures include blepharoptosis correction, lingual frenectomy, tympanografts and removal of lip haemangioma. Individuals with LDS1 and LDS2 required the most surgical interventions (online supplemental table 3).

\section{D photo facial surface analysis, $\mathrm{N}=39$}

The mean age was 17.9 years (range 2.4-57.4 years); 20 female and 20 male subjects. The $3 \mathrm{D}$ photos of five young patients ( $n=4 \mathrm{LDS}, \mathrm{n}=1 \mathrm{SGS}$ ) had to be excluded due to poor quality and lack of cooperation. The average of right and left measurements was used, since no significant soft tissue asymmetry was detected (figure 2, online supplemental table 4).

The cranial base width $(Z=-1.14)$, midface depth $(Z=-1.35)$ and lower face depth $(Z=-1.50)$ were reduced subclinically in all subgroups except for LDS5. These results indicate a tendency for midface and mandibular hypoplasia. Moreover, the cohort exhibited subclinical alterations in the vertical plane, including morphological face height $(Z=-1.54)$ and upper face height $(Z=-1.09)$, with the exception of LDS5. Intercanthal distance was subclinically increased $(Z=1.16)$. Nasal dimensions showed an increased subnasal width $(Z=1.20)$, while nasal projection $(Z=-1.01)$ and alar length $(Z=-1.38)$ were subclinically decreased. The dimensions of the lips were not significantly affected.

\section{LDS subtype distinctions}

SGS subtype demonstrated a more uniform but severe phenotype with a greater percentage of affected craniofacial measurements beyond $\pm 2.0 \mathrm{Z}$-score compared with the LDS subtypes. Lower facial projection $(Z=-2.79)$, total facial height $(Z=-3.14)$ and upper facial height $(Z=-4.30)$ were profoundly deficient in SGS, whereas intercanthal distance was significantly increased $(Z=3.13)$. In LDS1 and especially LDS2, there was a

A
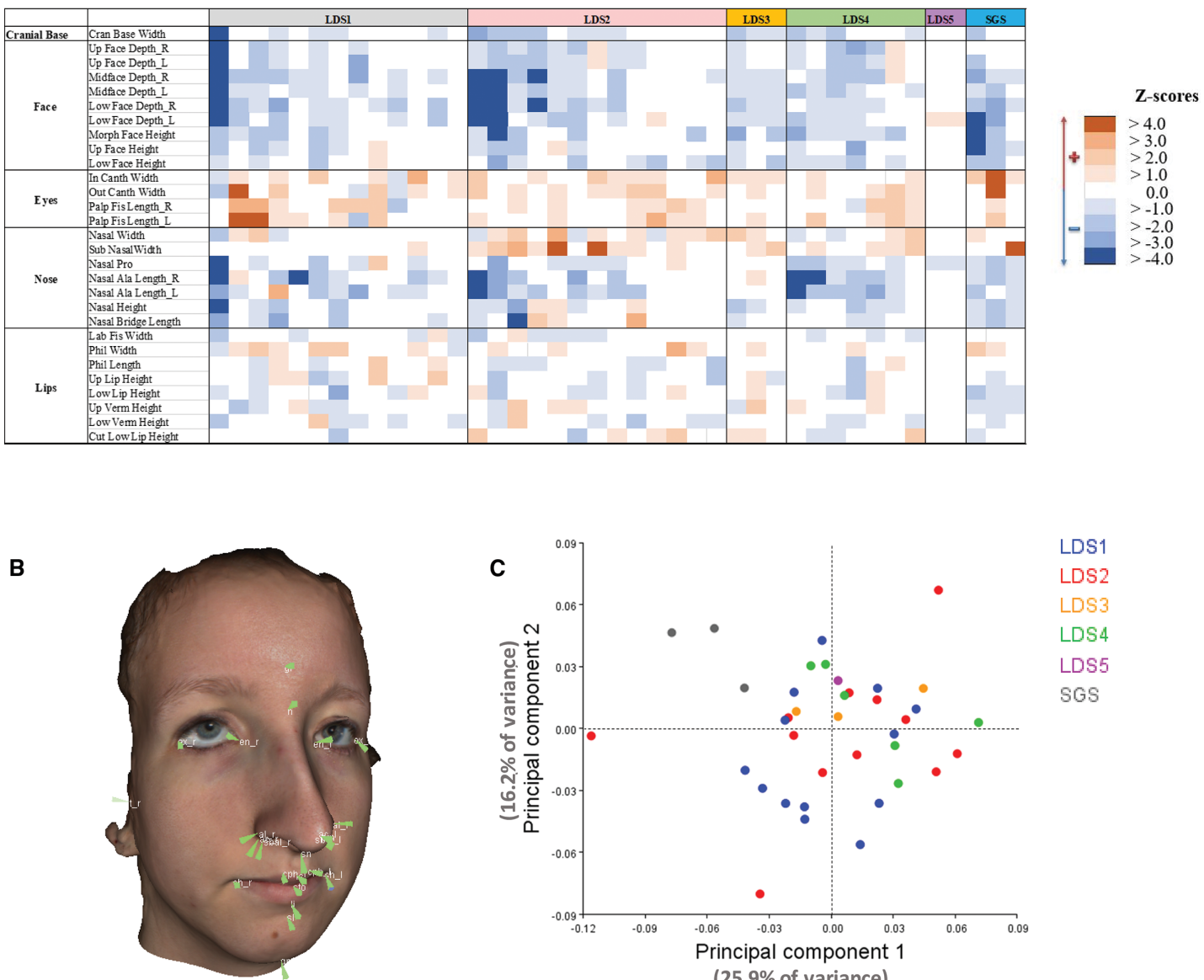

( $25.9 \%$ of variance)

Figure 2 (A) Heatmap of Z-scores depicting the deviation of the soft tissue measurements in the different LDS subtypes and SGS, based on the normal values provided by FaceBase. The orange shades represent positive values, blue represents negative values. SGS, LDS2 and LDS1 are the subtypes with extreme Z-score values. LDS2, followed by LDS1, have the greatest variability. Facial depth, eyes and nose projection measurements were uniformly affected across the cohort. The facial depth and nasal projection were the most negatively affected features, while the eye measurements were increased. (B) The 3D mesh view of the face of an individual with LDS2 with the annotated landmarks used for the photo surface analysis. (C) PCA plot of the 3D photo surface landmark coordinate data depicting the variability of the soft tissue morphology among the different LDS subtypes, with the greatest variability in LDS2. 3D, three-dimensional; LDS, Loeys-Dietz syndrome; PCA, principal component analysis; SGS, Sphrintzen-Goldberg syndrome. 


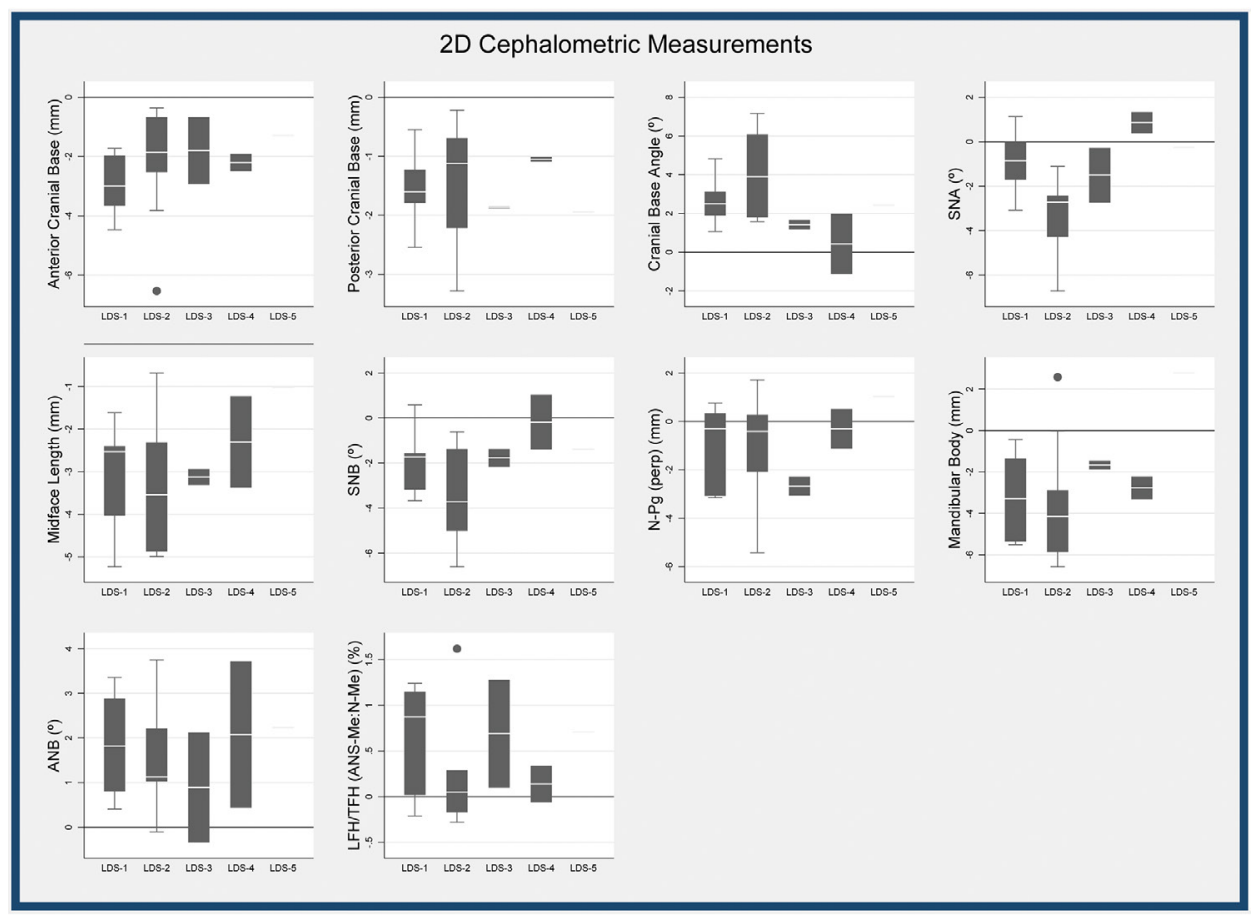

B

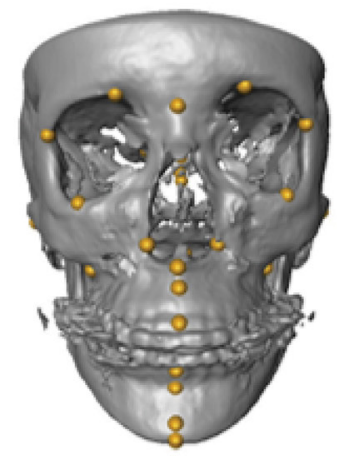

C

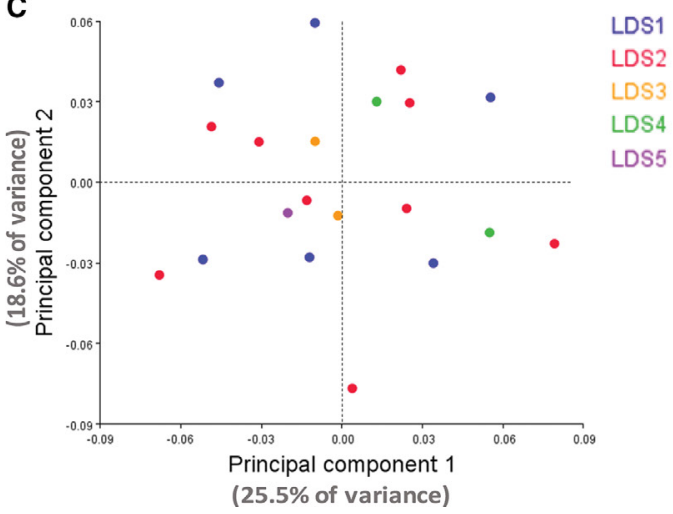

Figure 3 (A) Average Z-scores for each LDS subtype for each of the 2D cephalometric measurements, compared with normal values for the general population. The growth and position of the cranial base, maxilla and mandible are significantly affected in LDS, especially in LDS1 and LDS2. (B) The 3D craniofacial landmarks used for the cephalometric and geometric morphometric analysis annotated on a 3D view of a skull (C). PCA plot depicting the variation in the craniofacial shape in LDS. The variability in LDS2 and LDS1 can be appreciated, while all other subtypes are closer to the means of the axes. 2D, two-dimensional; 3D, three-dimensional; LDS, Loeys-Dietz syndrome; PCA, principal component analysis; SNA, sella-nasion-A point; SNB, sellanasion-B point.

characteristic heterogeneity, including patients who had normal $Z$-scores and others with Z-scores beyond \pm 2 . Therefore, the computed average Z-scores per LDS type were not representative for these two subtypes. In LDS3 and LDS4, there was less variability and no extreme Z-scores.

\section{Cephalometric analysis, $\mathrm{N}=20$, LDS only}

CBCT scans were obtained for 20 patients with LDS (10 female; 10 male); mean age was 26.9 years (9-57 years). No patients with SGS had CBCTs.

The mean cranial base angle (nasion-sella-articulare) was 3.00 SD above the norm, indicating a flattened cranial base angle, with the most significant alterations in LDS2 $(Z=4.20)$. Platybasia (defined as a cranial base angle $>143^{\circ}$ ) was detected in four cases among the LDS2 subtype (figure 3A). There was also significant shortening of the anterior cranial base (sella-nasion,
$\mathrm{Z}=-2.35$ ), with greatest deviation in the LDS1, LDS2 and LDS4. The posterior cranial base (sella-articulare, $Z=-1.56$ ) was subclinically affected (figure 3 , online supplemental table 5).

In the vertical plane, there was reduced anterior facial height (nasion-menton, $\mathrm{Z}=-2.45$ ) and posterior facial height (sellagonion, $Z=-1.95$ ). The lower face height, ramus height and gonial angle were within normal range.

In the sagittal plane, the midface length (condylion-A point, $\mathrm{Z}=-3.02$ ) was significantly reduced. The overall cohort mean Z-scores for SNA (sella-nasion-A point) and SNB (sella-nasion-B point) were -1.84 and -2.31 , respectively, with the most significant deviations in the patients with LDS2 (SNA, $\mathrm{Z}=-3.31 ; \mathrm{SNB}, \mathrm{Z}=-3.28$ ). Moreover, the mandibular body length was reduced in all LDS subtypes (gonion-pogonion, $Z=-2.83$ ), except for LDS5, where it was significantly increased $(Z=2.78)$. 
Overall, the craniofacial alterations from most severe to least severe were identified in LDS2, LDS1, LDS5, LDS3 and LDS4. The single patient with LDS5 had a broadly different craniofacial pattern than the other types, with normal cranial base and increased growth of the mandible (mandibular body length $=2.78$; lower facial height $=4.23$ ).

We tested for significant differences via ANOVA for each of the cephalometric measures across the three groups: LDS1, LDS2 and other LDS types. No statistically significant craniofacial cephalometric measures were found among the various LDS subtypes except for SNA, which was significantly smaller in LDS2 ( $\mathrm{p}<0.05$ for all comparisons), indicating significant maxillary hypoplasia.

\section{Pharyngeal airway assessment and hyoid bone position, $\mathrm{N}=20$, LDS only}

In $55.0 \%$ of the patients, there was reduced PAS. This included all six patients with LDS1, four LDS2, one LDS3 and one patient with LDS4 (online supplemental figure 1). The same patients had significantly reduced minimum CSA. The vertical position of the hyoid bone, based on the H-MP measurements, was low in $47.6 \%$ of the patients, many of whom also had reduced PAS.

\section{Multivariate analysis of 3D craniofacial shape: 3D photos and CBCT}

The PCA plot of the 3D photo data supported the findings of the cephalometric and 3D photo Z-score analyses and demonstrated the variability in LDS2. On the plot for the soft tissue landmark data (figure 2C), PC1 explained 25.9\% of the shape variance while PC2 explained an additional 16.2\%, for a total of $42.1 \%$ of the facial shape variance across the entire LDS cohort. LDS2 expanded across PC1 and PC2 axes in both directions, whereas the other LDS subtypes cluster closer to the centre of the plot. In contrast, SGS cluster separately and on the negative aspect of PC1 axis, indicating a clear morphological difference in these patients.

According to the PCA on the CBCT data, PC1 explained $25.5 \%$ of craniofacial shape variance and PC2 explained an additional $18.6 \%$, for a total of $44.1 \%$ of craniofacial shape variance (figure 3C). LDS1 and LDS2 cohorts spanned both the PC1 and PC2 axes, however, LDS2 had more extreme variability. LDS3 and LDS5 were closer to the centre of the plot on the negative PC1 axis, while LDS4 was on the positive PC1 axis.

\section{DISCUSSION}

This study is the first to quantitatively examine the craniofacial anomalies associated with two TGF- $\beta$-opathies, LDS and SGS, and to correlate the craniofacial phenotype to genotype using a multimodality approach. While the more severe craniofacial abnormalities in LDS have been previously reported, including craniosynostosis, hypertelorism, strabismus and cleft palate, these features were not common in this cohort compared with significant hypoplasia of the maxilla and mandible, abnormal eye shape with increased sclera show and blepharoptosis, abnormal lip shape, low-set ears and skin translucency, with more than $50 \%$ of the participants exhibiting these characteristics. Several of these features also indicate abnormalities of the cranial base.

The results of the quantitative cephalometric analysis conducted on CBCT images further revealed the underlying skeletal abnormalities and sources of dysmorphologies not readily identified with the clinical or surface analysis. Multiple angular measurements associated with the cranial base were significantly altered. The mean cranial base angle was over 3 SDs above the norm and four cases of platybasia were detected in LDS2. The length of the anterior cranial base was reduced. A more inferior location of the sella turcica contributed to an alteration in the inclination of the anterior cranial base, which resulted in an increased cranial base angle, increased SN-FH (Frankfort Horizontal plane) angle, and the extremely low SNA and SNB angles. The changes of the cranial base clearly impacted the development of the maxilla and mandible.

According to the clinical, cephalometric and 3D surface morphometric assessment, significant retrognathia of the mandible and maxilla was observed throughout the cohort. The cephalometric analysis confirmed that the retrognathia is not only due to positional changes of the cranial base but to the absolute underdevelopment of the maxilla and mandible. Vertically, there was a reduction in both anterior and posterior facial heights, but the cephalometric data suggest the cranial base and upper facial skeleton are the source of underdevelopment, as the ramus height and gonial angle were within the normal range.

Less clear are the underlying skeletal and developmental abnormalities contributing to the clefting of the uvula, although our data suggest a wider maxilla in the transverse plane in patients with LDS2 compared with the other types. Given the small sample size, we could not apply multiple testing corrections.

Using geometric morphometric analysis of overall facial shape, PCA analysis of both the CBCTs and 3D photos shows increased variability in LDS2, followed by variability in LDS4, with SGS clustering at the negative extreme of PC2. LDS1, LDS3 and LDS5 tend to cluster closer to the intersection of PC1 and PC2, suggesting these patients represent more of a mean LDS phenotype in terms of skeletal and facial shape. Although the sample size is small for the dimensionality of this multivariate geometric morphometric analysis, we are confident about the validity of these results given the consistent pattern of affected traits through clinical phenotyping and cephalometric analysis. In addition to LDS2 displaying the greatest phenotypic variability, the more severe abnormalities were identified in LDS2 and SGS, as confirmed by the craniofacial anomalies' indices. This suggests unique craniofacial developmental perturbations that are specific to TGFBR2 and SKI mutation variants.

The value of the $3 \mathrm{D}$ photos and surface analysis is rapid capture of information and the ability to digitally acquire anthropometric measurements. This is particularly valuable for assessing young and less cooperative patients while gathering quantifiable data. Additionally, we were able to detect subtle clinical feature variations, such as a relatively increased intercanthal distance, an increased nasal width, and decreased nasal projection and height, throughout the cohort. One of the limitations of this analysis is the limited normative values from FaceBase. Some normative data are based on very small groups with as little as six subjects, and some of the SD values are as low as 1.0 , which can lead to relatively increased Z-scores. In addition, there are no published 3D facial norms for individuals of non-European descent. These factors could potentially influence the validity of the generated Z-scores.

An important finding was the prevalence of TMD. This may be attributed to structural craniofacial abnormalities, such as the high percentage and severity of mandibular retrognathism, the underlying connective tissue disorder or a combination of the two factors. Some morphological changes were detected through qualitative CBCT assessment, but a more specialised analysis is required for further assessment that exceeds the scope of this paper. Interestingly, TMD has also been linked to a lower oral health-related quality of life in patients with LDS. ${ }^{15}$ An animal 
model may assist in understanding the TMD aetiology and development in LDS.

Our analysis revealed a tendency for the development of sleeprelated breathing disorders. OSA in LDS, to our knowledge, has only been described in one case report of a patient with LDS2. ${ }^{24}$ Apart from the 10 subjects who had a diagnosis of OSA, a larger number reported snoring $(n=17)$ and history of adenoidectomy $(n=9)$. Moreover, the quantitative analysis of CBCTs demonstrated a reduction in posterior airway space, low position of the hyoid, and a retrognathic mandible and maxilla, all of which are known contributors or risks for OSA. ${ }^{25-31}$ Considering the effect of OSA on cardiovascular events and hypertension, ${ }^{32}$ it is extremely important to consider sleep apnoea studies and assessment of craniofacial abnormalities in these patients.

One of the limits of this study and all studies of rare diseases is the small cohort and sample size. Additionally, we could not safely obtain CBCT images for all individuals due to the young age and radiation risks. Despite this, we have optimised the research potential by the deep clinical phenotyping and use of multimodality imaging analysis. Previous studies on craniofacial dysmorphology have relied on 'gestalt' and limited clinical assessment. And while 'gestalt' is helpful for obvious features and diseases with homogeneous characteristics, it is less useful when there is significant phenotypic variation within a cohort. Unfortunately, such assessments do not provide methods to link the craniofacial developmental aberrations to the underlying mutations, even when the genotype is established. The strength of this study is the multimodality morphometrics, particularly the skeletal imaging, which provides an objective, quantitative phenotypic characterisation and demonstrates the segregation of craniofacial development to the mutations along the TGF- $\beta$ pathway. Clearly, the mutations associated with the TGFBR2 and SKI genes have a profound effect on craniofacial development with perturbations associated with the cranium and cranial base with severe downstream effects on the orbit, maxilla and mandible. Additionally, the highest variability noted in LDS2 suggests epigenetic, non-canonical or distant enhancers of the TGFBR2 gene mutation that may induce the variable phenotypic spectrum. We continue to examine these variations in the mouse models of TGF- $\beta$ pathway mutations, however, the opportunity to deeply phenotype and understand the variation in human subjects is invaluable.

In summary, as precision medicine becomes more possible, the detailed data provided by the present study will be added to the growing literature and will assist clinicians in the future in predicting the clinical anomalies per genetic variant. In addition, we address for the first time the higher prevalence of TMD and relatively increased risk of OSA in the case of LDS, particularly in the LDS1 and LDS2 subtypes, and encourage clinicians to include more detailed assessments regarding these conditions as part of the physical evaluation of these patients.

\section{Twitter Priyam Jani @priyamjani}

Acknowledgements We thank the personnel of the NIDCR Dental Clinic who were involved in the collection of the data and the patients who participated in the study.

Contributors Conceptualisation—JSL. Methodology—KA, DKL and PJ. Formal analysis - KA, DKL and CK, Resources — JSL and PAF-G. Data curation-KA, DKL, PJ, CK and RM. Writing (original draft) preparation-KA. Writing (review and editing)JSL, DKL, PJ, RM and PAF-G. Visualization—KA and PJ. Supervision—JSL. Funding acquisition -JSL and PAF-G. Patient recruitment and consent acquisition-PO and NC. All authors reviewed the manuscript critically for important intellectual content and approved the version to be published.

Funding This research was funded by the Intramural Research Program of the National Institute of Dental and Craniofacial Research (PI: JSL; DDS, MD, FACS, ZIA
DE000746 04 and ZID DE000728 10) and in part by the National Institute of Allery and Infectious Diseases (PI: PAF-G, ZIA AI001203 04).

Competing interests None declared.

\section{Patient consent for publication Obtained.}

Ethics approval The main study and the collaborated study protocols were approved by the Institutional Review Boards at their respective institute ( ClinicalTrials.gov numbers, NCT02639312 for the Natural History of Craniofacial Anomalies and Developmental Growth Variants Study and NCT02504853 for the Natural History and Genetics of Food Allergy and Related Conditions Study). A signed informed consent was obtained from all the participating patients and/or their guardians.

Provenance and peer review Not commissioned; externally peer reviewed.

Data availability statement Data are available upon reasonable request. All data relevant to the study are included in the article or uploaded as supplemental information.

Supplemental material This content has been supplied by the author(s). It has not been vetted by BMJ Publishing Group Limited (BMJ) and may not have been peer-reviewed. Any opinions or recommendations discussed are solely those of the author(s) and are not endorsed by BMJ. BMJ disclaims all liability and responsibility arising from any reliance placed on the content. Where the content includes any translated material, BMJ does not warrant the accuracy and reliability of the translations (including but not limited to local regulations, clinical guidelines, terminology, drug names and drug dosages), and is not responsible for any error and/or omissions arising from translation and adaptation or otherwise.

Open access This is an open access article distributed in accordance with the Creative Commons Attribution Non Commercial (CC BY-NC 4.0) license, which permits others to distribute, remix, adapt, build upon this work non-commercially, and license their derivative works on different terms, provided the original work is properly cited, appropriate credit is given, any changes made indicated, and the use is non-commercial. See: http://creativecommons.org/licenses/by-nc/4.0/.

\section{ORCID iDs}

Konstantinia Almpani http://orcid.org/0000-0001-7558-6796

Priyam Jani http://orcid.org/0000-0001-7768-7865

\section{REFERENCES}

1 Loeys BL, Chen J, Neptune ER, Judge DP, Podowski M, Holm T, Meyers J, Leitch CC, Katsanis N, Sharifi N, Xu FL, Myers LA, Spevak PJ, Cameron DE, De Backer J, Hellemans J, Chen Y, Davis EC, Webb CL, Kress W, Coucke P, Rifkin DB, De Paepe AM, Dietz HC. A syndrome of altered cardiovascular, craniofacial, neurocognitive and skeletal development caused by mutations in TGFBR1 or Tgfbr2. Nat Genet 2005;37:275-81.

2 Bertoli-Avella AM, Gillis E, Morisaki H, Verhagen JMA, de Graaf BM, van de Beek G, Gallo E, Kruithof BPT, Venselaar H, Myers LA, Laga S, Doyle AJ, Oswald G, van Cappellen GWA, Yamanaka I, van der Helm RM, Beverloo B, de Klein A, Pardo L, Lammens M, Evers C, Devriendt K, Dumoulein M, Timmermans J, Bruggenwirth HT, Verheijen F, Rodrigus I, Baynam G, Kempers M, Saenen J, Van Craenenbroeck EM, Minatoya K, Matsukawa R, Tsukube T, Kubo N, Hofstra R, Goumans MJ, Bekkers JA Roos-Hesselink JW, van de Laar IMBH, Dietz HC, Van Laer L, Morisaki T, Wessels MW, Loeys BL. Mutations in a TGF- $\beta$ ligand, TGFB3, cause syndromic aortic aneurysms and dissections. J Am Coll Cardiol 2015;65:1324-36.

3 Doyle AJ, Doyle JJ, Bessling SL, Maragh S, Lindsay ME, Schepers D, Gillis E, Mortier G, Homfray T, Sauls K, Norris RA, Huso ND, Leahy D, Mohr DW, Caulfield MJ, Scott AF, Destrée A, Hennekam RC, Arn PH, Curry CJ, Van Laer L, McCallion AS, Loeys BL, Dietz HC. Mutations in the TGF- $\beta$ repressor Ski cause Shprintzen-Goldberg syndrome with aortic aneurysm. Nat Genet 2012;44:1249-54

4 Robinson PN, Neumann LM, Demuth S, Enders H, Jung U, König R, Mitulla B, Müller D Muschke P, Pfeiffer L, Prager B, Somer M, Tinschert S. Shprintzen-Goldberg syndrome: fourteen new patients and a clinical analysis. Am J Med Genet A 2005;135:251-62.

5 Carmignac V, Thevenon J, Adès L, Callewaert B, Julia S, Thauvin-Robinet C, Gueneau L, Courcet J-B, Lopez E, Holman K, Renard M, Plauchu H, Plessis G, De Backer J, Child A, Arno G, Duplomb L, Callier P, Aral B, Vabres P, Gigot N, Arbustini E, Grasso M, Robinson PN, Goizet C, Baumann C, Di Rocco M, Sanchez Del Pozo J, Huet F, Jondeau G, Collod-Beroud G, Beroud C, Amiel J, Cormier-Daire V, Rivière J-B, Boileau C, De Paepe A, Faivre L. In-Frame mutations in exon 1 of Ski cause dominant ShprintzenGoldberg syndrome. Am J Hum Genet 2012;91:950-7.

6 Loeys BL, Schwarze U, Holm T, Callewaert BL, Thomas GH, Pannu H, De Backer JF, Oswald GL, Symoens S, Manouvrier S, Roberts AE, Faravelli F, Greco MA, Pyeritz RE, Milewicz DM, Coucke PJ, Cameron DE, Braverman AC, Byers PH, De Paepe AM, Dietz HC. Aneurysm syndromes caused by mutations in the TGF-beta receptor. N Engl I Med 2006;355:788-98.

7 Akutsu K, Morisaki H, Takeshita S, Sakamoto S, Tamori Y, Yoshimuta T, Yokoyama N, Nonogi H, Ogino H, Morisaki T. Phenotypic heterogeneity of Marfan-like connective 
tissue disorders associated with mutations in the transforming growth factor-beta receptor genes. Circ J 2007;71:1305-9.

8 Seo GH, Kim Y-M, Kang E, Kim G-H, Seo E-J, Lee BH, Choi J-H, Yoo H-W. The phenotypic heterogeneity of patients with Marfan-related disorders and their variant spectrums. Medicine 2018;97:e10767.

9 Loeys BL, Chen J, Neptune ER, Judge DP, Podowski M, Holm T, Meyers J, Leitch CC, Katsanis N, Sharifi N, Xu FL, Myers LA, Spevak PJ, Cameron DE, De Backer J, Hellemans J, Chen Y, Davis EC, Webb CL, Kress W, Coucke P, Rifkin DB, De Paepe AM, Dietz HC. A syndrome of altered cardiovascular, craniofacial, neurocognitive and skeletal development caused by mutations in TGFBR1 or Tgfbr2. Nat Genet 2005;37:275:275-81.

10 Tug E, Loeys B, De Paepe A, Aydin H, Gideroglu K. A Turkish patient of typical LoeysDietz syndrome with a Tgfbr2 mutation. Genet Couns 2010;21:225-32.

11 Ting TW, Lai AHM, Choo JTL, Tan TH. Loeys-Dietz syndrome in a Southeast Asian Hospital: a case series. Eur J Pediatr 2014;173:387-91.

12 Camerota L, Ritelli M, Wischmeijer A, Majore S, Cinquina V, Fortugno P, Monetta R, Gigante L, Sangiuolo FC, Novelli G, Colombi M, Brancati F. Genotypic categorization of Loeys-Dietz syndrome based on 24 novel families and literature data. Genes 2019;10:E764

13 Van Hemelrijk C, Renard M, Loeys B. The Loeys-Dietz syndrome: an update for the clinician. Curr Opin Cardiol 2010;25:546-51.

14 Jani P, Nguyen QC, Almpani K, Keyvanfar C, Mishra R, Liberton D, Orzechowski P, Frischmeyer-Guerrerio PA, Duverger O, Lee JS. Severity of oro-dental anomalies in Loeys-Dietz syndrome segregates by gene mutation. J Med Genet 2020;57:699-707.

15 Nguyen QC, Duverger O, Mishra R, Mitnik GL, Jani P, Frischmeyer-Guerrerio PA, Lee JS. Oral health-related quality of life in Loeys-Dietz syndrome, a rare connective tissue disorder: an observational cohort study. Orphanet J Rare Dis 2019;14:291.

16 Liberton DK, Mishra R, Beach M, Raznahan A, Gahl WA, Manoli I, Lee JS. Comparison of three-dimensional surface imaging systems using landmark analysis. J Craniofac Surg 2019;30:1869-72.

17 Cignoni P, Callieri M, Corsini M, Dellepiane M, Ganovelli F, Ranzuglia G. MeshLab: an open-source mesh processing tool. Sixth Eurographics Italian Chapter Conference, 2008:129-36.

18 Weinberg SM, Raffensperger ZD, Kesterke MJ, Heike CL, Cunningham ML, Hecht JT, Kau CH, Murray JC, Wehby GL, Moreno LM, Marazita ML. The 3D facial norms database: Part 1. A web-based craniofacial anthropometric and image repository for the clinical and research community. Cleft Palate Craniofac J 2016;53:185-97.
19 Adams DC, Collyer ML. Sherratt E: geomorph: software for geometric morphometric analyses. R package version 2.1.x, 2015.

20 Liberton DK, Verma P, Contratto A, Lee JS. Development and validation of novel threedimensional craniofacial landmarks on cone-beam computed tomography scans. J Craniofac Surg 2019;30:e611-5.

21 Adams DC, Otárola-Castillo E, Paradis E. geomorph: an r package for the collection and analysis of geometric morphometric shape data. Methods Ecol Evol 2013:4:393-9.

22 Klingenberg CP. MorphoJ: an integrated software package for geometric morphometrics. Mol Ecol Resour 2011;11:353-7.

23 Harris PA, Taylor R, Thielke R, Payne J, Gonzalez N, Conde JG. Research electronic data capture (REDCap)--a metadata-driven methodology and workflow process for providing translational research informatics support. J Biomed Inform 2009;42:377-81

24 Takenouchi T, Saito H, Maruoka R, Oishi N, Torii C, Maeda J, Takahashi T, Kosaki K. Severe obstructive sleep apnea in Loeys-Dietz syndrome successfully treated using continuous positive airway pressure. Am J Med Genet A 2013;161A:1733-6.

25 Neelapu BC, Kharbanda OP, Sardana HK, Balachandran R, Sardana V, Kapoor P, Gupta A, Vasamsetti S. Craniofacial and upper airway morphology in adult obstructive sleep apnea patients: a systematic review and meta-analysis of cephalometric studies. Sleep Med Rev 2017;31:79-90.

26 Jamieson A, Guilleminault C, Partinen M, Quera-Salva MA. Obstructive sleep apneic patients have craniomandibular abnormalities. Sleep 1986;9:469-77.

27 Maltais F, Carrier G, Cormier Y, Sériès F. Cephalometric measurements in snorers, nonsnorers, and patients with sleep apnoea. Thorax 1991;46:419-23.

28 Johal A, Patel SI, Battagel JM. The relationship between craniofacial anatomy and obstructive sleep apnoea: a case-controlled study. J Sleep Res 2007;16:319-26.

29 Partinen M, Guilleminault C, Quera-Salva MA, Jamieson A. Obstructive sleep apnea and cephalometric roentgenograms. The role of anatomic upper airway abnormalities in the definition of abnormal breathing during sleep. Chest 1988:93:1199-205.

30 Sutherland K, Lee RWW, Cistulli PA. Obesity and craniofacial structure as risk factors for obstructive sleep apnoea: impact of ethnicity. Respirology 2012;17:213-22.

31 Cistulli PA, Sullivan CE. Sleep apnea in Marfan's syndrome. Increased upper airway collapsibility during sleep. Chest 1995:108:631-5.

32 Peppard PE, Young T, Palta M, Skatrud J. Prospective study of the association between sleep-disordered breathing and hypertension. N Engl J Med 2000:342:1378-84. 